\title{
Place de la métrologie au service du contrôle de qualité du matériel industriel utilisé au laboratoire d'analyse biologique du Centre de Transfusion Sanguine d'Oujda au Maroc
}

\author{
Khalid EL BEKKAYE et Zaina SIDQI
}

CRTS Oujda, Maroc

\begin{abstract}
This work is an initiation into the knowledge of the installed metrology there is 10 years at the level of blood transfusion centers, so it plays an important role at the level of the qualification of the material used in the laboratory of medical analysis, in the production laboratory of the blood by-products, and at the level of the devices of storage, preservation and transport of the blood products. Indeed the safety of unstable blood product in a blood transfusion center is bound to the quality of its preparation by using a calibrated material and in compliance with the standard of use, where from a quality control is a requirement which must be periodically applied according to an annual program, to correct its anomalies. Devices and measuring instruments used in the centers of transfusion are controlled with a variable periodicity of one in four times a year according to the procedure of qualification by the persons in charge of metrology, approximately 279 devices and instruments are controlled in the center of transfusion of Oujda in 2014, among which more than $80 \%$ Activities of the center are reserved for the storage and the preservation of the unstable blood products, with a $95 \%$ conformity.
\end{abstract}

\section{Introduction}

La sécurité d'un produit tel que le produit sanguin labile dans un centre de transfusion sanguine est liée à la qualité de sa préparation en utilisant un matériel calibré et conforme à la norme d'utilisation, d'où le contrôle de qualité des appareils est une exigence qui doit être appliquée périodiquement selon un programme annuel, afin de corriger toute anomalie détectée . Une mauvaise manipulation peut coûter une vie humaine.

\section{Définitions}

\subsection{La métrologie}

C'est l'outil de la qualité, c'est la science de la mesure intégrant l'ensemble des connaissances et des actions permettant d'attribuer à une propriété particulière des objets, matériaux ou phénomènes [2-6]

Sa valeur exprimée en nombre, elle permet d'exprimer une grandeur par un symbole qui s'appuie sur la théorie de la mesure : Grandeur = mesure $\times$ unité.
Son but : obtenir des mesures fiables

\section{La fonction métrologie recouvre :}

- La création de dossiers d'achat; La réception;

- La mise en service ;

- La création et la tenue des documents techniques relatifs au matériel;

- La formalisation des conditions d'utilisation ;

- La coordination;

- Le traitement des anomalies de fonctionnement et l'organisation de la maintenance ;

- Le suivi de l'inventaire et de l'état du parc d'équipement du service.

\subsection{La maintenance}

La maintenance regroupe les actions de dépannage et de réparation, de réglage, de révision, de contrôle et de vérification des équipements matériels (Appareil, objets manufacturés, etc.) ou même immatériels (logiciels).

La maintenance doit prendre en considération de nombreuses contraintes comme la qualité, la sécurité, l'environnement, le coût... [8-10].

\subsubsection{La maintenance corrective,}

Qui consiste à intervenir sur un équipement une fois que celui-ci est défaillant. Elle se subdivise en :

${ }^{a}$ khalid_elbekkaye@hotmail.fr 
○ Maintenance palliative : dépannage (donc provisoire) de l'équipement, permettant à celui-ci d'assurer tout ou partie d'une fonction requise ; elle doit toutefois être suivie d'une action curative dans les plus brefs délais.

○ Maintenance curative : réparation (donc durable) consistant en une remise en l'état initial.

\subsubsection{La maintenance préventive,}

Qui consiste à prévenir la panne, pour des raisons de sûreté de fonctionnement (le dépoussiérage, le nettoyage, la lubrification et protection)

\subsection{La matériovigilance}

concoure à assurer une veille sanitaire par un processus continu de recueil, d'enregistrement, d'identification, de traitement, d'évaluation et d'investigation des événements indésirables liés à l'utilisation des dispositifs médicaux afin d'optimiser leur sécurité d'emploi, actuelle et future [9] .

\section{Objectifs}

$>$ Promouvoir le système qualité en métrologie au Centre Régional de Transfusion Sanguine d'Oujda (CRTSO).

> S'intégrer à la stratégie nationale en assurant la continuité des activités déjà acquises tout en essayant d'améliorer le processus de métrologie, et participer aux audits de qualité.

Corriger les anomalies relevées au cours des audits précédents

$>$ Améliorer la recherche et la connaissance de la métrologie par la rénovation du domaine sanitaire et par la participation aux congrès, séminaires et audits au cours de l'année.

$>$ Suivi de maintenance curative instantanée et préventive programmée des appareils du CRTSO.

\section{Matériels et méthodes}

Les appareils et instruments de mesures utilisés au centre de transfusion sont contrôlés avec une périodicité variable de un à quatre fois par an selon la procédure de qualification par le responsable de métrologie, ainsi le matériel utilisé pour contrôle varie selon le type d'appareil :

$>$ Les masses étalon ADAM WS004A et WS0010B pour contrôler la fiabilité, la justesse-exactitude et l'excentration des balances selon les procédures du référentiel [5-7]

$>$ Thermomètre de référence étalonné TESTO 950 pour contrôler les thermomètres, et le système Cox tracer et eviSENSE pour suivi et contrôle des températures relevés des appareils de la chaine de froid selon les techniques du processus [1-3].
Un tachymètre TESTO 465 étalonné pour contrôler la vitesse de centrifugation.

\section{Résultats}

Les appareils et instruments de mesures utilisés au centre régional de transfusion d'Oujda sont contrôlés avec une périodicité variable de un à quatre fois par an selon la procédure de qualification par les responsables de métrologie, environ 279 qualifications des appareils et instruments contrôlés au centre de transfusion d'Oujda en 2014, dont plus de $80 \%$ sont réservés au stockage et conservation des produits sanguins labiles, avec un taux de conformité de $95 \%$.

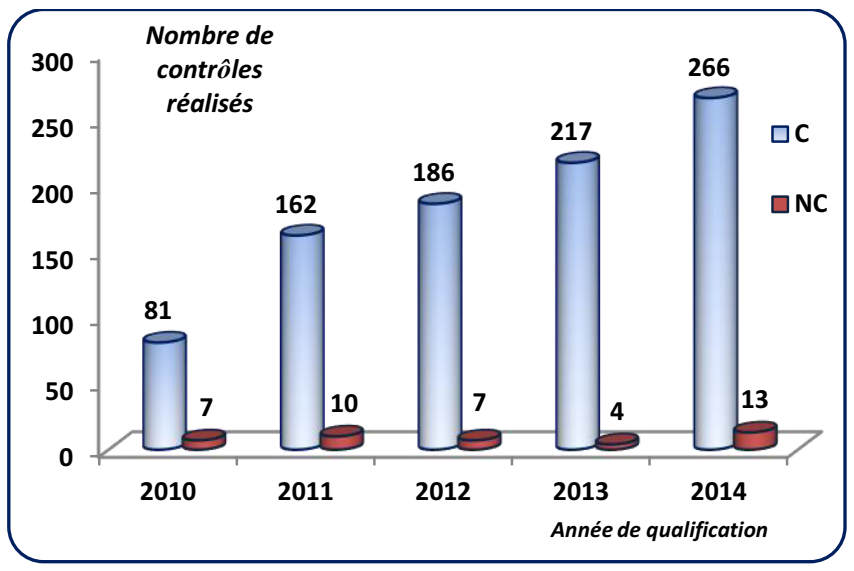

Figure 1: Appareils qualifiés depuis 2010 à 2014 (C: conforme, $\mathrm{NC}:$ non conforme)

Le nombre de donneur par collecte a augmenté depuis 2010 jusqu'au 2014, ce qui a nécessité l'utilisation de plus d'appareils, et donc le nombre de qualifications a proportionnellement augmenté, le résultat de conformité est très satisfaisant.

Le résultat du contrôle est soit exploité sous forme d'un fichier Excel, Word ou graphique accompagné par une conclusion qui va confirmer le critère d'acceptation :

$\rightarrow$ Conforme.

$\rightarrow$ Non-conforme

$\rightarrow$ Réservé (conditions particulières d'utilisation)

La détermination de conformité d'un appareil, est un indicateur de performance permettant d'assurer à l'efficacité d'utilisation du matériel conformément à une norme 


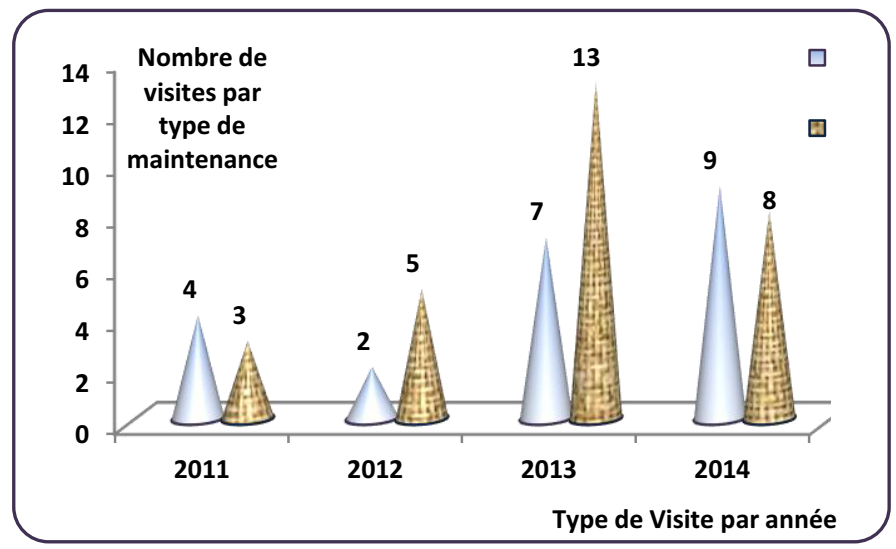

Figure 2 : Maintenance des appareils (2010- 2014) (MP: maintenance préventive ; MC : maintenance curative)

La maintenance préventive et curative des appareils est accordée aux sociétés externes par contrat, ce qui a permis de prévenir leurs pannes, et d'écarter les non-conformités relevés durant leurs usages.

La visite préventive des appareils est trimestrielle, par société de maintenance, ainsi, le nombre de visites varie d'une année à l'autre selon les objectifs et accords définis dans le contrat, par contre la visite curative est maintenu après chaque panne de l'appareil, ainsi on remarque que les visites curatives ont augmenté, ceci est lié à la durée de vie des appareils (vieillissement), au nombre d'examens effectués (augmentation), parfois d'autres paramètres interviennent tels que l'environnement et conditions de travail (eau distillée, tension du courant électrique, les rayons lumineux, local ...), les utilisateurs, et l'emplacement des appareils.

\section{Discussions}

Le suivi des indicateurs de performance [11] en métrologie et en maintenance est essentiel dans la bonne conduite d'une démarche qualité et doivent être visibles et accessibles à tous, et permettant à une accréditation du laboratoire [4].

Mais avant tout, il faut lister ceux dont on a besoin et structurer leur relation avec le système documentaire. Parmi ceux-ci, le plus important est la fiche de vie de l'équipement qui assure une traçabilité complète des actions effectuées ou des évènements survenus.

Ainsi, la bonne gestion du matériel médicauxtechniques nécessitant:

- la collaboration de l'ensemble des acteurs

- La connaissance et l'application stricte des consignes: d'utilisation, d'entretien et des conduites à tenir en cas de panne, d'où la nécessité d'intégrer la formation continue en métrologie dans la plan d'action annuel.
- La mise à jour chaque fois que nécessaire de la documentation relative au matériel

\subsection{Les utilisateurs restent toujours responsables}

- Du respect des consignes d'utilisation,

- De l'entretien lié à l'usage et en particulier du nettoyage,

- De la mise à jour des documents d'utilisation lorsqu'ils existent,

- Du signalement immédiat des anomalies de fonctionnement.

Les utilisateurs coordonnent la réalisation des opérations de vérification et d'étalonnage. Ils en contrôlent le planning et les enregistrements.

5.2 Mettre en place un système de suivi dans le temps de ces équipements de mesure consiste à :

$>\quad$ Ouvrir les fiches de vie des équipements, $>\quad$ Définir la périodicité de suivi,

$>\quad$ Étalonner / vérifier périodiquement les équipements,

E Établir les procédures de métrologie «Qualification, conditions d'utilisation, de maintenance et de nettoyage »

$>$ Maîtriser les paramètres d'environnement,

$>\quad$ Enregistrer les résultats d'étalonnages ou vérifications,

$>\quad$ Assurer la traçabilité et le raccordement des étalons

$>\quad$ Évaluer les sous-traitants métrologiques

\section{Conclusion}

Le nombre de qualifications est en augmentation continue depuis l'installation du système assurance qualité et la disposition du matériel de qualification au centre régional de transfusion sanguine d'Oujda. La conformité obtenue lors de qualification des appareils rassure une bonne maintenance et gestion du matériels médicaux-techniques et prouve une continuité d'approvisionnement, raisonnable en matériel soumis à un contrat de maintenance, et une disponibilité en ressources humaines qualifiés et habilités.

\section{Références}

1. A-A. Novarti ; Manuel de gestion, maintenance et utilisation du matériel de la chaîne du froid pour le sang, sang et produits sanguins sécurisés, OMS (2008)

2. A. Mazzei (2009) ; La Métrologie, le Dossier, N¹ IMQ 
3. CNTSH : Référentiel des Bonnes Pratiques Transfusionnelles (2009) ; Rabat Maroc ; p17

4. Critères spécifiques pour l'accréditation des laboratoires d'étalonnage et d'essais sur site, document AL212, révision 00-06 (2006),

M.I.C.E ; NM ISO 17025, Maroc

5. D. Louvel ; Procédure pour la vérification des balances et d'enceintes climatiques et thermostatiques (1998), rapport d'une commission mixte ASFILAB/SESTP France Pharma pratiques 8 (6) 500-518

6. Guide Métrologie, Radiometer analytical,( 2009), Germany ; DOC $\mathbf{1 8 2 . 7 7}$

7. Incertitude de mesure : évaluation en atelier, janvier-février (2002), TECHNOLOGIE 117, 59-61

8. J. Roboh, Production maintenance, $\mathbf{N}^{\circ} \mathbf{3 4}$, dossier technologies MRJ. Paris (2011)

9. La Matériovigilance, (2005), eurasanté, p23

10. Manuel d'entretien et de maintenance des appareils de laboratoire OMS (2008)

11. M. Pillet, C-M et P. Bonnefous, A. Courtois, Gestion de production : les fondamentaux et les bonne pratiques (2012) EYROLLES, Paris, p341 